\title{
The Social Organization of Neonatal Nurses' Feeding Work
}

\author{
Catherine Ringham, $P h D, B S N, R N, C N e o N(C) \odot$ \\ Janet Rankin, PhD, RN \\ Lenora Marcellus, PhD, RN
}

Catherine Ringham, Janet Rankin, and Lenora Marcellus have no conflicts of interest to declare.

Funding. The author(s) received no specific grant or financial support for the research, authorship, and/or publication of this article.

\begin{abstract}
Purpose: We report findings from an institutional ethnography (IE) of nurses' work of feeding infants within an increasingly technical organization of NICUs.

Sample: Five primary informants; 18 secondary informants.

Design: The institutional ethnographic approach included field observations, interviews, and phone and e-mail conversations. Our analysis followed accounts of what actually happened within the textual organization of nurses' work.

Main Outcome: Nurses' feeding practices are directed by protocols that arise within multiple documentation systems and clinical technologies. These systems produce barriers to nurses' efforts to skillfully feed infants.

Results: Prioritization of quality and safety perspectives can obscure and constrain the ordinary yet critical clinical reasoning neonatal nurses employ during feeding work. Clinical technologies that have been developed to improve safety can paradoxically disrupt the ability of nurses to respond in the moment to neonatal feeding cues. This finding provides nurses, leaders, and policymakers with insight into why policies and procedures may not be followed as expected.
\end{abstract}

Keywords: NICU; feeding; nurses’ work; institutional ethnography; standards; QI

$\mathrm{F}$ OR NeONATAL NURSES, MANAGING THE increasingly complex technologies that have been developed to support high-acuity patients has rendered the "basic" work of infant feeding as less urgent than responding to computerized devices and their alarms. Systematic attention to quality and safety has also placed a great deal of focus for clinical teams on the concept of risk and practices needed to mitigate potential for error. ${ }^{1-3}$ Electronic medical records, ventilators, automated drug dispensing technology, barcoded systems for expressed breast milk (EBM), and infusion pumps are designed with a central interest in safety and risk management. Each of these technologies incorporate features designed to address factors seen as contributing to human error and demand a particular kind of nursing attention. ${ }^{4-6}$ For example, features such as alarms and "time stamped" processes hold nurses to embedded record-keeping measures designed to ensure and document safe, error-free patient care.

Premature infants have unique feeding needs. ${ }^{4,5}$ Evidence supports the use of holistic, 
infant-led, cue-based feeding practices that contribute to positive feeding experiences for preterm infants and their longterm relationship with food..$^{6-8}$ The capacity of care providers and parents to read and act on subtle infant cues supports the physiologic development of organized sucking, swallowing, and breathing (SSB) necessary for successful oral feeding skills. ${ }^{9} 10$ However, nurses' attention is frequently redirected in ways that disrupt less-visible feeding work. In this article we report the findings from an institutional ethnography (IE) of nurses' work of feeding infants within an increasingly technical organization of NICUs. We describe how feeding arises as a problematic feature of the very technical NICU environment and investigate some of the cue-based feeding strategies that have been implemented in the NICU. We analyze how feeding has been standardized within evidence-based protocols and discuss how standardized clinical practice documents compete with the ordinary demands of nursing work. We conclude that the fundamental core of nearly all patient care in the NICU is the ordinary, hands-on work of attending to infants' needs and cues within the rhythm of their scheduled feeding.

\section{FEEDING MATTERS}

Best feeding practices are underpinned by evidence-based guidelines and protocols that have been widely introduced into NICU nurses' work. ${ }^{10-13}$ Infant feeding is the nucleus of patient care in the NICU. Infants learn to feed and to suckle, and parents learn how to support their fragile infants to feed, grow, and develop neurologically. ${ }^{10}$ Gaining the skill and strength to sustain oral feeding can be slow and arduous for preterm infants, and it requires nurses and parents to pay painstaking attention to each infants' subtle and overt cues. $^{14,15}$ Although feeding is the hub of everyday NICU work, other priorities often draw nurses' attention away from important activities linked to feeding, including observing and responding to hunger and feeding cues, preparing milk, and teaching mothers about breast and/or formula feeding. Nurses know that positive feeding experiences impact infants' development and parents' ability to manage feeding problems after discharge from the NICU. ${ }^{8,10,16}$

\section{TECHNOLOGY, EVIDENCE, AND \\ STANDARDIZED FEEDING PROTOCOLS}

Contemporary neonatal care is largely organized by rigorous appraisal frameworks (such as those that accompany the Cochrane pyramid for knowledge ${ }^{17}$ ) and the application of best evidence to practice that are the result of scientific advances. New evidence-informed technologies and protocols to direct feeding practices and to support nurses' knowledge are an important feature of clinical practice. ${ }^{12,13,18}$ As well, the proliferation of charts and flow sheets where nurses document their adherence to protocols absorb nurses' time and effort. Of course, what is also important, but less institutionally visible, is nurses' day-to-day decision making that is based on practical, experiential knowledge. It is this knowledge that our research indicates is being disruptedboth by the rapid introduction of numerous, targeted quality improvement (QI) initiatives and the textual systems for recording and auditing nurses' compliance. Nurses' capacity to respond to what is happening in their moment-to-moment patient care is succumbing to a burgeoning of standardizing care practices that are being monitored and appraised for quality and safety.

\section{METHODS}

Institutional ethnography is a qualitative research approach with critical feminist underpinnings designed to investigate power relations and uncover how power structures organize how people do their work. ${ }^{19}$ IE was developed by sociologist Dorothy Smith to make visible tensions occurring in peoples' lives, and to raise awareness of inequities that arise from power relations and dominant forms of knowledge. In this study, neonatal nurses provide a critical position to understand what they do and what they say as expert knowers of the work they do and the setting they are in. The aim is to ethnographically describe nurses' work (and its tensions) and then to map or trace those points of strain into the health system to show how rules and ordinary administrative processes intersect with their work practices.

Institutional ethnography draws on broad institutional practices, such as policymaking and workforce planning, that coordinate and organize how people's work is directed and represented in written and computerized formats. Important data sources in an IE inquiry are the written, electronic, material documents referred to as texts. ${ }^{20,21}$ Many texts accompany and intersect with people and their work, including chart forms, clinical practice guidelines, electronic health records, infusion pumps with standardized drug libraries, and so forth. The investigative exercise is to discover how texts are used in the settings and the kinds of practices they direct. The researcher tracks how texts move beyond the local setting, how they are developed, and how they are used by other people who rely on them to generate the representative facts upon which to make decisions. ${ }^{22}$ In this research, conducted from the research standpoint of NICU nurses' direct care practices, the clinical documents, policies, and chart forms were an essential contribution to the empirical data. They were used in conjunction with observations and interviews to show how written materials entered and directed NICU nurses' work processes. For further explanation of IE please refer to Smith's text, Institutional Ethnography: A Sociology for People. ${ }^{19}$

\section{Participants}

Five primary informant neonatal nurses providing direct patient care in an urban, Level 2 NICU in Alberta, Canada, volunteered to participate. They had a wide range of experience working in a NICU setting, from three years to more than 20. Observations and interviews with primary informants allowed us to reliably describe how nurses' work in a 
Level 2 NICU routinely unfolds. In IE, the test of whether the primary informant "sample" is representative is whether the descriptions "ring true" with other people who work in similar settings. ${ }^{23}$ We anticipate that NICU nurse readers will find the described process of NICU nurses' work familiar-it is work that goes on commonly during the care of infants in Level 2 NICUs. Eighteen secondary informants included other point-of-care nurses, nurse leaders, managers, unit clerks, public health nurses, and parents of preterm infants admitted to a Level 2 NICU. The secondary informants were purposefully recruited during the early analysis of the primary informant data. They were sought for what they could tell us about how their work intersected with the primary informants' work processes. In IE, the research "sample" effectively provides a "slice" of institutional practices that are used to show how primary informants' practices are being institutionally coordinated. Pseudonyms were used for all participants. Ethical approval for the study was obtained from the Conjoint Health Research Ethics Board (CHREB) (REB13-1194).

\section{Data Collection}

Field observations (with field notes) and one-to-one semi-structured interviews were conducted with primary informants by Catherine Ringham (CR). Observations, completed over three to five hours of each nurses' shift, were an opportunity to see nurses' work in motion, while follow-up interviews allowed for clarification of work processes and interconnections with the work of others. Interviews were digitally recorded and transcribed by CR. Primary informants' activities were traced to intersections with secondary informants and institutional texts and processes. The accounts of primary and secondary informants' and descriptions of their various actions and activities provided data from which we drew our threads of analysis. Note that as analysis proceeds in IE, we no longer refer to our informants as primary and secondary, but simply as "informants" who provide clues into the institutional coordination of their work.

\section{Analysis}

Consistent with the IE method, we focused on identifying and describing those occasions when nurses' decisions and actions seemed incongruent with what the situation may have warranted; for example, those occasions when they may have been pulled in two or more directions and were required to prioritize among competing demands. CR did the initial analytic work to expose these disjunctures. Discussion with the team occurred in person and by e-mail to further tease out the essential lines of inquiry. We questioned the nurses about these instances, and we used these junctures of their work as points of departure into our investigation. It was through this analytical process that nurses' work of feeding infants arose as a problematic feature of the nurses' everyday practices. This led us to our deeper analysis of texts, field notes, and transcribed interviews. Analysis was an iterative process of reading transcripts and field notes, writing, and reading again to allow the critical threads of analysis to surface. We followed nurses' work into institutional processes that organize how nurses' work unfolds and made visible organizational rules and regulations that link back into the coordination of nurses' everyday work that do not necessarily "fit" with what actually happens.

\section{RESULTS}

Points of tension were identified in nurses' work when they were feeding infants. We explore five disjunctures and explicate where such tensions arise from informants' experiences with protocols, guidelines, documentation, and feeding work; fitting knowledge about the feed into the documentary record; aligning nurses "knowledge of their patient with feeding protocols; the subordination of nurses" feeding work; and what actually happens in feeding work.

\section{Protocols/Guidelines/Documentation and Feeding Work}

The health region where this research was conducted used two texts to guide health care providers in feeding preterm infants. One was a physician ordered feeding protocol called "Safe Individualized Nipple-feeding Competence" (SINC). ${ }^{13}$ The other was titled the "Regional Oral Feeding Guideline" (ROFG). ${ }^{18}$ As a QI project SINC was intended to address what was viewed as (specifically) nurses' "random approach to feeding that may contribute to poor short-term and longterm clinical outcomes." ${ }^{13}$ At the time of data collection SINC was designed to benefit moderately preterm infants (30-32 weeks' gestation) with respiratory morbidity. At the discretion of (and order from) a physician, the protocol requires nurses to follow a precisely organized, stepwise progression of feeding intervals and calculated volumes of milk. The strategy proposed to enhance preterm infants' oral-feeding skill and competency.

The ROFG guides nurses to closely observe infant characteristics that communicate effective SSB coordination and endurance but does not explicitly direct milk volumes or time at breast or bottle. A physician orders a daily total fluid intake (TFI) and the nurse calculates the volume for each oral feed based on intravenous (IV) fluid intake that also must be considered in the total. Nurses can use more discretion within the wide parameters of the guideline's direction for percentage of total TFI and mode of intake-breast, bottle, or gavage. For example, an infant at ROFG "Stage 2" (of three broad stages) may take a volume between 10 percent and more than 80 percent of their daily volume by breast or bottle nipple. Beyond the given parameters and supporting evidence, nurses rely on their critical and practical knowledge of each infant and their ongoing clinical assessment of the infant's capacity and skill to feed successfully. They observe the infant and determine when the remaining calculated volume of milk should be infused via a gastric tube and feeding pump. 
Informants suggested that the ROFG was a guideline "with more flexibility" than SINC, allowing nurses to make most decisions regarding feeding tolerance and progression. One nurse commented that "feeding was essentially in the hands of nurses" with the ROFG. Yet they reportedly have limited discretion with SINC, which is much more prescriptive in regard to how the volume of milk for each feed is to be divided between nipple and nasogastric tube feedings. Regardless whether an infant is demonstrating feeding cues and is able to retain a strong suck-swallow-breathe pattern, nurses said that SINC instructs them to stop the bottle feed (or have the mother stop breastfeeding) once the infant has suckled the prescribed volume. Informants pointed out that quantity is difficult to precisely measure with breastfeeding; nurses and mothers must guess how much the infant might have taken. The remaining calculated daily volume of milk for each feed is given by nasogastric tube. For infants who do not meet the criteria to be on the SINC protocol, nurses are expected to follow the ROFG.

\section{Fitting Knowledge About the Feed into the Documentary Record}

The prescriptive directions written into SINC and the ROFG play out in the actual work of nurses in a much less straightforward manner than either of the documents capture. Watching and recording nurses "doing" their feeding work makes the work appear routine and easy, but the observations belie what is actually happening during each feed. Karen provided a glimpse into her complex feeding knowledge when she explained how important it is to read infants' cues and pay close attention to infant position during each feed:

[I] place the baby on a pillow, on their side and then carefully pace the feed by tipping the nipple to one side or removing it every few sucks. This gives the baby an opportunity to stay organized with their suck/swallow coordination and go at their own speed.
The careful pacing that Karen describes is not easily transformed into procedural directions. Nor is it easy to capture within the clinical record-the document used by nurses and others to identify where the infant is in relation to the SINC stages and guided advances in the ROFG. The nurses' documentation record (Figures 1 and 2) in the setting where the research was conducted was also being used to audit how well the SINC protocol was being followed. Adherence was determined by the data nurses entered into the feeding record. Figure 1 shows the layout of boxes for recording information. However, the predetermined categories in the feeding flow sheet constrain what nurses can enter. The form cannot accommodate descriptions of particular feeding strategies or full descriptions of an infant's behavioral responses. The flow sheet limits descriptions of how nurses make adjustments to the way they hold or pace an infant during a feed. Paying careful attention to an infant's cues can result in more competent feeding skills, which means that nurses will advance the infant to the next stage of the protocol or guideline.

Fiona described tension between her feeding work and what she was required to document. She explained how she records feeding data on the paper flow sheet, a form that also requires notations about the required, timed checks of IV fluids, medications, and safety equipment. She then enters these data again, into the electronic record. The records are organized with numerical codes that are aligned with a legend. The legend has six coded strategies that the nurse can indicate were employed during the feeding session. Figure 2 shows the legend with the associated codes to be entered into the boxes in Figure 1.

Despite the codes and the legend, Fiona told us how the flow sheet "lacks the space for any explanation about how the feed actually went." She elaborated by detailing how she had assessed the infant's prefeed cues. The infant's cues suggested to Fiona that she "offer $5 \mathrm{~mL}$ in an open nipple rather than using a bottle." The legend (Figure 2) had

FIGURE 1 - Nurses' documentation form.

Patient ID \#

\begin{tabular}{|c|c|c|c|c|c|c|c|c|c|c|c|c|c|c|c|c|c|c|c|c|c|}
\hline \multirow[b]{2}{*}{$\stackrel{\Xi}{\xi}$} & \multirow[b]{2}{*}{ 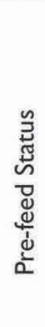 } & \multicolumn{3}{|c|}{ Breast Feed } & \multicolumn{5}{|c|}{ Tube Feed } & \multicolumn{4}{|c|}{ Bottle Feed } & \multirow[b]{2}{*}{ 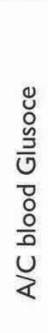 } & \multirow[b]{2}{*}{ 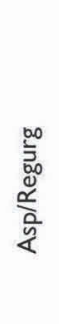 } & \multirow[b]{2}{*}{ Additives } & \multirow[b]{2}{*}{ 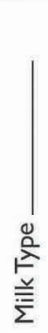 } & \multirow[b]{2}{*}{$\begin{array}{l}\text { 을 } \\
\text { 을 }\end{array}$} & \multicolumn{3}{|c|}{ Output } \\
\hline & & 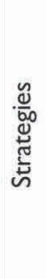 & 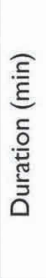 & 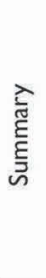 & 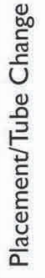 & 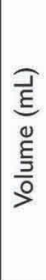 & 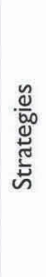 & 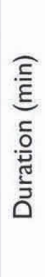 & 齐 & 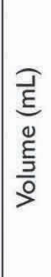 & 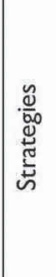 & 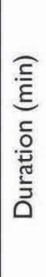 & 离 & & & & & & 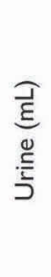 & $\begin{array}{l}\text { Urine, SG, } \\
\mathrm{pH}, \text { gluc, } \\
\text { bld, prot } \\
\text { OR } \\
\text { Other } \\
\text { output }\end{array}$ & $\begin{array}{l}\bar{\delta} \\
\text { Dे }\end{array}$ \\
\hline & & & & & & & & & & & & & & & & & & & & & \\
\hline & & & & & & & & & & & & & & & & & & & & & \\
\hline & & & & & & & & & & & & & & & & & & & & & \\
\hline
\end{tabular}


FIGURE 2 Legend for nurses' documentation form.

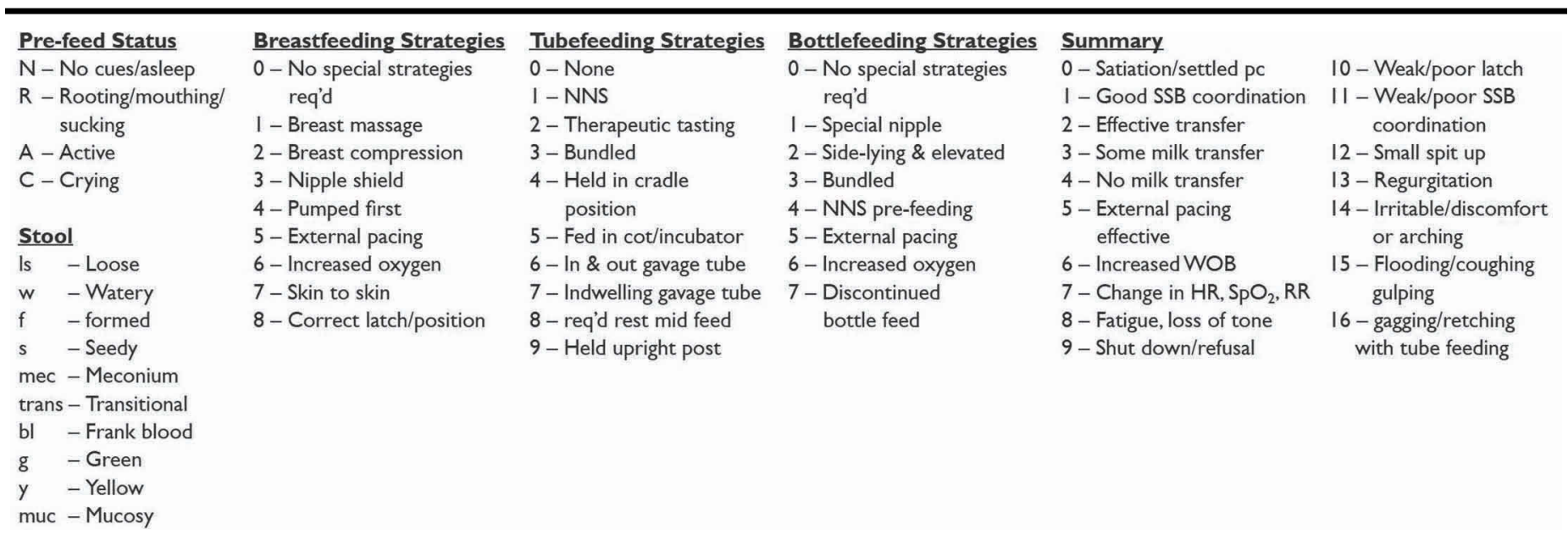

Abbreviations: $\mathrm{HR}=$ heart rate; NNS = non-nutritive sucking; RR = respiratory rate; SSB = sucking, swallowing, and breathing; $\mathrm{WOB}=$ work of breathing.

limited choices and the capacity of the small boxes was inadequate, but she said, "I just make [what I have done] fit." Fiona went on to say:

People [referring to doctors and nurses] don't look at the full picture, so babies get moved up a stage without seeing what the baby is actually doing overall, not just with feeding but also having bradys and desats, bloodwork, tolerating baths and other "procedures."

Fiona is describing a few of the multitude of priorities that she balances in the interests of the infant, but which appear as separate, unrelated entities when recorded on the flow sheet.

The flow sheet is audited regularly by nurse practitioners (NPs) who do QI projects and evaluate practice and patient outcomes. Fiona reported that the NPs assess whether the SINC protocol is followed consistently and assessments of feeding behavior and responses are recorded appropriately. However, reducing complex feeding activities into codified categories restricts how nurses communicate the various aspects of individual feeding behavior and responses. The disconnect between what is recorded and what actually happens is magnified when the data in the flow sheet are accepted as "facts." The nurses' entries on the flow sheet are used to show evidence of ongoing QI work but are not a comprehensive description of what happened during each feed. A text that is intended to represent what is going on leaves behind a great deal of what occurred. Nonetheless, nurses' efforts to fit their feeding work into textual (institutional) categories is aggregated and used to make broad claims and decisions about how nurses feed infants, and then this can be linked into patient outcomes. This "textual hyperreality" 24 becomes the authorized knowledge about what is going on in the feeding practices with preterm infants in the NICU, and it is used to make claims about improvements.

\section{Aligning Nurses' Knowledge of Their Patient with Feeding Protocols}

It is not only the flow sheet that nurses "make fit" into what they need to do. Our informants explained how they also make efforts to adapt the SINC protocol to fit each context of practice. Karen explained:

I kind of follow what SINC suggests, to start with a small amount of milk in an open nipple. She may only take $2 \mathrm{mLs}$, then spit the nipple out. That's an indication she has had enough. We have to watch and figure it out for each baby. There really is no recipe.

Karen was describing her work with infant Samera, who she said was "too old" for SINC-Samera did not fit the age parameters for the protocol. Karen clarified that although SINC is an official feeding regime only for certain kinds of infants, she "found it helpful" to guide her decision making around feeding even if the infant did not fit the official criteria. Karen's knowledge goes well beyond the directions in the SINC protocol or the ROFG, which she apparently chose not to use. She knows that it is important to read infants' cues and pay close attention to how the infant is positioned, and so forth. Feeding, for nurses, is much more complicated than mere timing and volumes.

The cue-based practices that nurses have learned require them to closely attend to the infant's body language. This is work that does not easily align with the emphasis on ordered percentage of volumes and maximum minutes for breast or open nipple techniques now prescribed in SINC. The ROFG stresses infants' "emerging readiness cues" and provides directions for "non-nutritive sucking," "therapeutic tasting," "pairing tube feeding with breast feeding," and "slow flow nipples." Nurses have this theoretical knowledge that they translate into skillful practice; ultimately though, neither the ROFG nor SINC protocol tell them how to feed the infant. This nursing know-how is tacit and particular, a complex 
synthesis of nurses' knowledge and experience. Theoretically, the ROFG and SINC protocol introduce different levels of authority into nurses' work; one guides practice, while the other is a physician's order. Our data begin to show how these levels of authority are always mediated by nurses' knowledge and that nurses must include what they know about the infant and whatever else is going on in the unit.

\section{The Subordination of Nurses' Feeding Work}

Infants need to feed regularly. The skill and experience that feeding requires drew our analytic interest. We also noted how nurses seemed to gloss over and underplay their feeding expertise and knowledge. As indicated in the data excerpt below, feeding is considered so mundane as to be boring:

To be honest, I find this kind of nursing work [feeding] kind of boring compared to what I used to do. But it is so important! If we push these kids, they can develop oral aversion and shut down or they will choke because they are not coordinated enough. And then you have a whole bunch of other problems to deal with - that makes it hard for parents later on. You have to know this stuff, or you can really cause problems.

In contrast to this characterization by one of our informants, another informant described in great detail her adeptness with knowing how to position and read each infant's cues, explaining:

With a fragile feeder, they need to be set up properly for the feed. In other words, we gently wake them if they are not already stirring. We take their warm milk and with the baby on a pillow on our lap, we place the baby on their side with the nurse's hand supporting the top of the baby's shoulders and neck. We have to help them to take breaks in sucking if they forget to breathe. This all takes time, and care.

The importance, but also the routine nature, of feeding work is clear in these nurses' descriptions of their experiences.

\section{What Actually Happens in Feeding Work}

Despite how the previous sections reveal what nurses know about the complexity of observing, positioning, and responding to "fragile feeders," and despite the precise directions for feeding that SINC and the ROFG have introduced to feeding, feeding does not happen according to "best practices." Repeatedly during the observations, nurses interrupted an infant's feed to attend to other things going on. One informant described an occasion when:

\footnotetext{
After I started the feed, I had to put the kid in the cot, walk over to the other side of the pod and check the med on the syringe pump with the other nurse; it was very disruptive. The baby lost the flow of the feed. Five times I got up to answer the alarm on the pump!!! I felt like I was cueing the med, not the baby!
}

Another nurse described a similar experience:

I was the second checker for a med. And I had this baby who was on SINC . . a fragile feeder. The baby needed my full attention so I could monitor and read his cues. I was up and down so many times during that feed. It took me 50 minutes to complete it . . that is not supposed to happen!

Expanding our investigation beyond the texts expected to govern feeding (SINC and ROFG) we discovered institutional "rules" that competed with feeding. Most often these were quality and safety practices related to computerized equipment. Our data described how digitalized, preprogrammed alarms consistently undermined the low tech, hands-on care of infants-particularly the work of feeding, as we show in the previous informant accounts. Responding to equipment seemed to be a proxy for attending to the physical presence of the actual infant in the cot. In contrast to the insistent mechanical alarms, infant's cries could not generate focused responses from nurses who were occupied with answering the pump alarms and checking medications.

\section{Medication Delivery}

It was not only the intrusive alarms that coordinated what nurses attended to. Karen explained how a QI project, designed to reduce the incidence of medication errors for IV medication administration, established standard drug dosing and concentration, infusion pumps, and procedures. Karen outlined the number of extra steps that must be included in the process of checking the medications and flushing the IV tubing at several points during the administration of IV medications. Nurse informants reported that the process is complex and time consuming and they are constantly being pulled away from each infant's care to adhere to medication safety practices. One nurse explained:

It is almost impossible to get through a feed without having to put the baby down and answer the pump alarm for a med. Sometimes there is no one around to come do the check... what are we supposed to do then?

Under these conditions, nurses' capacity to focus on feeding was constantly diverted. The protocols driven by electronic and computerized technology competed for nurses' attention. What nurses know about feeding infants and infants' needs were subordinated to the powerful demands of the technology.

\section{Safe Handling of EBM}

Paradoxically, the safety procedures for handling EBM also interfered with nurses' knowledge about what fragile feeders needed from them. The EBM tracking systems introduced in NICUs absorbed a great deal of nurses' time. Fiona described receiving EBM from a mother who had arrived to the NICU to feed her infant. The infant was awake and moving his hands to his face and making sucking movements with 
mouth - cues suggesting he was hungry and needed to be fed. Fiona described how:

Before I could get the baby ready for the feed, I had to check all the bottles the mother brought into the tracking system, label them, then mix the additives with the milk to feed to the baby, label the bottle with a new label (that gets printed out on the printer over there), then go through the process of scanning the bottle again before pouring the right amount into a new bottle, labeling it, scanning the baby's ID label in the cot, and then scanning that bottle as "ready to feed." I also have to go to the fridge [at the other end of the unit], get the baby's tray of milk, sort the new and old bottles, check every label for the date and time pumped, and organize what needs to be put into the freezer and what needs to be thrown out. It all has to be done properly and quickly so the milk isn't left out of the fridge for too long. And you have to get the baby fed.

When asked about how she knew that she needed to follow these steps, Fiona referenced the "Safe Handling and Administration of EBM" policy, which she told us was a "level one policy," the highest level of administrative edict in the Health Service. Fiona explained the various levels of policy and their authority:

There are guidelines and policies for feeding; the EBM one is a policy, so we have to follow it. The ROFG guides what we do and SINC is somewhere in between.

Fiona's understanding about the importance of the procedural integrity of the EBM practices was underscored by NICU leaders who were responsible for auditing the system, a process to track noncompliant nurses and generate a monthly report for NICU staff.

Thus, in the instance that Fiona was describing, although the infant was awake and showing cues to feed, and despite that Fiona knew the milk had come from his mother, Fiona delayed her work to support the mother/infant feeding experience in order to "safely" handle the EBM. She prioritized registering and labeling the bottles of EBM to comply with the policy. This delay did not make sense for the mother, who could not proceed without Fiona, or for the infant who was at risk for fatiguing and generating functional over-stimulation that results in profound sleepiness and neurological disengagement; the predictable physiologic responses that reduce an infant's capacity to competently suck, breathe, and swallow.

A nurse activating the EBM policy in another instance described working with a mother who, after having breastfed until her infant tired, was pumping her breast milk at the infant's bedside for the "top up" feeding through the tube. The nurse described:

The infant's mother handed me a bottle of her freshly pumped milk. Before I could administer the EBM, I had to put the milk in the tracking system as "received," label the bottle, and then place the correct amount in another bottle, label it and scan the label to "prepare feed." I had seen the mother pump the milk into a bottle and knew it came from her, and I also knew the breastmilk was for her infant who was in the cot beside her. And I knew that the fresh milk was far better than any refrigerated, reheated milk.

What the nurse knew to be safe and risk-free administration of the EBM in this situation (she witnessed the milk and its origin and had no doubt that the mother intended it for her infant) was at odds with the institutional processes that required specific tracking, labeling, and checkpoints. The risk of disease transmission through EBM is not zero, but it is very low. ${ }^{25}$ However, the handling and administration of EBM, having been targeted as a region-wide QI initiative, now takes up considerable nursing time and is being subject to routine audits.

\section{DISCUSSION}

While the observations and quotes from informants seem straightforward and unremarkable, our analysis builds on these foundational accounts. We describe nurses mediating their feeding work with their other work, such as medication administration, their work with parents, response to urgent situations, and their work with guidelines, policies, and protocols. We show how the preponderance of procedural directions that have been introduced into NICU culminate in tensions and contradictions that undermine how feeding proceeds. Contrary to infant feeding science that points to the importance of observing infant cues, the data from this research show how nurses' attention to an infant's suckling during a nipple feed is organized to take a low priority.

Despite the scientific evidence underpinning the feeding protocols and guidelines, routine accomplishment of responsive, uninterrupted feeding is rarely possible. Nurses' knowledgeable actions are overruled by pervasive systems designed to navigate institutional risk and improve the quality and safety of patient care. The double-check requirement at every stage of the medication infusion process, and the required barcode checks related to computerized tracking and administering EBM we have described here, are among many systems and institutional processes dealt with by nurses. The analysis being explicated in this study is how, despite nurses' theoretical knowledge of current feeding science and despite (or, we suggest, perhaps even because of) so many disconnected clinical protocols and policies, nurses are unable to observe and respond to infants' cues. Our research showed that feeding is often rushed, interrupted, and truncated. Moreover, our research suggests that the layers of texts that are being used to move the new feeding science into everyday practices generate other barriers for skillful feeding practices. The paradigm of procedural, stepwise edicts is not well aligned with what infants and their mothers need from NICU nurses.

In the milieu of activity illustrated in nurses' accounts, infants were viewed "out of the corner of nurses' eyes," socially organized by technocratic influences to the periphery 
of nurses' full attention. Our data showed that while a central feature of neonatal nurses' everyday work is feeding infants, the particular activities inherent in how feeding actually happens are consistently subordinated to the dominant power relations embedded in the growing number of QI technologies and institutional policy and processes-even those designed to address feeding. Benzies and colleagues suggest that the constantly changing NICU environment adds to the complexity of everyday care for health care providers, infants, and families in an already complex health care system. ${ }^{26}$ With an IE analysis our work expands on the notion of the complexity of everyday nurses' work within health systems and builds an argument that the proliferation of guidelines and procedures and the primacy of policy frameworks override nurses' tacit and discretionary knowledge, thus creating fragmented care. This has serious consequences for effectively feeding infants in NICU.

Technologies provide useful advances in NICU nursing and, more specifically, feeding work. Indeed, programmable feeding pumps used for gavage feeding help nurses meet infants' nutritional needs when an infant is unable to take necessary nutrition by nipple. Using barcode technologies adds to the assurance that the right infant receives the right EBM. There is also no doubt that IV pump technologies improve medication safety. The issues we raise rest in how nurses' decisions in any given moment are organized toward adhering to protocols, orders, and computerized alarms that do not (and cannot) accommodate what nurses know about what is happening, not only with infant's physical cues but the many other contingencies that arise in the actual setting that constitute the "social practices" of NICU care.

When nurses' work is mechanistically separated into discrete work processes, it creates a problem-as though medication work, EBM handling, gavaging, feeding, teaching, assessment, communication, and documentary work can be extracted, examined, controlled, and reinserted into the ongoing activities. The technologies that are being purchased, serviced, and updated are introduced as advances that address identified problems such as "workflow," risk mitigation, and better patient outcomes. However, they often have not taken nursing knowledge and practice activities into account in their development and implementation. Our analysis develops a critique about how innovations are ideologically organized within understandings of "patient safety" and grounded in audits that generate calculative metrics; they are practices that we contend are a narrow construction of what counts as evidence or risk.

Both EBM and IV medications have been subject to QI technologies intended to reduce risk. It seems to make sense when taken in isolation from other aspects of nurses' work. Ethnographic descriptions of nurses at work generate clues for us to track and map into the dominant logic of QI, to learn more about how it actually plays out in the work of nurses. While nurses can describe and lament the delays and interruptions in what Karen described as the "arduous processes" in which complex feeding work happens, nurses do not have an analysis of how their work is being organized within broader institutional interests in ways that, we argue, are troubling.

We examined the paper and computerized texts nurses activated in their work processes which informed how we observed what happened in practice, and then examined the documents and computer fields to investigate how NICU nurses' work was organized. Our analysis included learning about how the health region's systems vet, classify, and systematize the governance of regulatory safety and QI texts that are introduced into clinical settings. We discovered that the policies, protocols, and guidelines being used in NICU were each generated independently, during different time frames, and often by different people or groups of people. In particular, this was the case with the feeding documents (the SINC protocol, the ROFG, and the EBM policy). Indeed, with the implementation of the SINC protocol, the use of the ROFG seemed to migrate away from nurses' view. The two sets of directions for feeding did not easily integrate with one another or with the unique needs of each infant, or the responsibilities of nurses' work as a whole. Disparate practices and processes generated new demands and use of nurses' time.

Our research findings raise a cautionary alert to the strong trend toward introducing segregated regulatory standards and technological alarms and stops for nursing tasks that require nurses to detach each task from the rest of what is going on in their nursing work. Can or should nurses' work be continuously improved by breaking it into discrete responsibilities that are to be monitored and accounted-as if they are not connected to any other action? Evidence from our research suggests that nurses' adherence to the built-in capacity of clinical technologies to both direct and scrutinize what they are doing undermines the necessary discretion and connectedness that human to human work requires.

The ethnographic data revealed nurses making considerable efforts to comply with the clinical standards established within the growing systems. However, our observations also found that it is common for nurses to cut corners with documentation, and to cosign one another's work retroactivelyrepairing the documentary record as though the work had unfolded in the procedurally correct way. On such occasions, despite nurses' intentions, they necessarily adapt or subvert the "official" rules in the best interests of patient care. Such noncompliance with clinical standards of practice is an ongoing issue for nurse managers and educators. One manager interviewed for this research expressed concern about the number of procedural errors identified during an audit of the EBM system. She insisted that if nurses would just "slow down" they would not make the procedural errors the audit had revealed. However, nurses' capacity to work more slowly is simply not possible within the rush that accompanies tightening work demands as acuity increases and nurses experience increasing challenges to improve efficiencies.

This research draws attention to the now taken-for-granted (and growing) presence of multiple technologies/procedural practices and QI initiatives that intersect directly with infant 
care and nurses' work. The technologies are imprinted with the established logic of risks, safety procedures, texts, scrutiny, and audits that are representative of QI initiatives. The hyperreality generated by people's work with texts and documentation is the foundational knowledge that is being used to manage nurses' work. Our research raises questions about the critical aspects of nurses' knowledge and expertise that is being usurped by institutionally driven rules.

\section{Limitations}

This study traces a single thread of connected practices that became central to the investigation. There were many other threads that could have illuminated how the health care “institution" embeds tensions into NICU nurses' work. In this paper, we have shown only a slice of the power relations that coordinate nurses' decisions about feeding. In addition, the research was limited to observations in only one NICU and our "institutional" interviews were limited to a particular geographic regional health authority. This constrains what we can say about NICU nursing more generally. Despite this limitation, we traced the texts and activities from nurses' accounts of their work into extended national and international neonatal initiatives that impact geographically dispersed NICUs. Thus, we are confident in our claim that we have uncovered a generalizing trend in the ruling practices that create tensions in neonatal nursing.

\section{CONCLUSION}

Current approaches to QI and patient safety may inadvertently override critical, practical nursing knowledge and activities that are needed in the best interests of infants. The knowledge being pushed aside is not only nurses' knowledge gained from being "in the moment," but it also includes a breadth of knowledge acquired from academic learning through coursework in neonatal specialty and unit orientation programs. Nurses' knowledge (especially what novice nurses know about their work) is being increasingly coordinated within a specter of the computerized texts like the electronic health record, and everchanging complex technologies embedded in NICU equipment. It is a trend that undermines how nurses might otherwise use the science of feeding. Standardizing feeding science with a focus on tools for safety and QI seems to inhibit nurses' ability to concurrently mediate their own knowledge and reduce interruptions during infant feeding. Nurses' wisdom and expert judgment, the very qualities that underpin the contributions of a professional nursing workforce, are being subordinated to standardizing accountability circuits that direct and then represent practice. We contend that while many QI initiatives have reduced risks of serious error, comprehensive improvements in the quality of nursing care are not being realized. Rather, what may be happening is a series of conceptual ideas about safe, quality care that undermine the extensive professional contributions nurses make in NICUs.

The feeding tensions that our ethnographic observations unveiled are tracked into the social organization of the NICU policies and guidelines. Our findings are an empirical explication of the coordinated, institutionally sanctioned quality and safety initiatives that undermine nurses' capacity to actually do cue-based feeding.

\section{ACKNOWLEDGMENTS}

Dr. Ringham acknowledges Dr. Carol Ewashen and Dr. Shahirose Premji for their contributions to the doctoral work from which the manuscript arises.

\section{REFERENCES}

1. Allen D. The importance, challenges and prospects of taking work practices into account for healthcare quality improvement. J Health Organ Manag. 2016;30(4):672-689. https://doi.org/10.1108/ JHOM-04-2014-0062

2. Lawless ST. The impact of continuous quality improvement on a system's healthcare costs. Pediatrics. 2016;137(4):1. https://doi.org/10.1542/ peds.2015-4164

3. Lyle-Edrosolo G, Waxman KT. Aligning healthcare safety and quality competencies: Quality and Safety Education for Nurses (QSEN), the Joint Commission, and American Nurses Credentialing Center (ANCC) Magnet ${ }^{\circledR}$ Standards Crosswalk. Nurse Lead. 2016;14(1):70-75. https:// doi.org/10.1016/j.mnl.2015.08.005

4. Shaker CS. Cue-based feeding in the NICU: using the infant's communication as a guide. Neonatal Netw. 2013;32(6):404. https:// doi.org/10.1891/0730-0832.32.6.404

5. Wellington A, Perlman JM. Infant-driven feeding in premature infants: a quality improvement project. Arch Dis Child-Fetal Neonatal Ed. 2015;100:F495-F500. https://doi.org/10.1136/ archdischild-2015-308296

6. Briere CE, McGrath J, Cong X, Cusson R. State of the science: a contemporary review of feeding readiness in the preterm infant. $J$ Perinat Neonat Nur. 2014;28(1):51. https://doi.org/10.1097/ JPN.0000000000000011

7. Fontana C, Menis C, Pesenti N, et al. Effects of early intervention on feeding behavior in preterm infants: a randomized controlled trial. Early Hum Dev. 2018;121:15-20. https://doi.org/10.1016/ j.earlhumdev.2018.04.016

8. John HB, Padankatti SM, Kuruvilla KA, Rebekah G, Rajapandian E. Effectiveness of oral motor stimulation administered by mothers of preterm infants-A pilot study. J Neonatal Nurs. 2018. https://doi.org/ 10.1016/j.jnn.2018.05.001

9. Jones LR. Oral feeding readiness in the neonatal intensive care unit. Neonatal Netw. 2012;31(3):148-155. https://doi.org/ 10.1891/0730-0832.31.3.148

10. Sables-Baus S, DeSanto K, Henderson S, et al. Infant-directed oral feeding for premature and critically ill hospitalized infants: guideline for practice. National Association of Neonatal Nurses; 2013.

11. Gelfer P, McCarthy A, Spruill CT. Infant driven feeding for preterm infants: Learning through experience. Newborn Infant Nurs Rev. 2015;15(2):64-67. https://doi.org/10.1053/j.nainr.2015.04.004

12. Lasby K, Dressler-Mund D. Making the literature palatable at the bedside: reference poster promotes oral feeding best practice. $A d v$ Neonatal Care. 2011;11(1):17-24. https://doi.org/10.1097/ANC. 0b013e318206elb4

13. Dalgleish S, Kostecky L, Blachly N. Eating in "SINC": safe individualized nipple-feeding competence, a quality improvement project to explore infant-driven oral feeding for very premature infants requiring noninvasive respiratory support. Neonatal Netw. 2016;35(4):217. https://doi.org/10.1891/0730-0832.35.4.217 
14. Giannì ML, Sannino P, Bezze E, et al. Does parental involvement affect the development of feeding skills in preterm infants? A prospective study. Early Hum Dev. 2016;103:123-128. https://doi.org/10.1016/ j.earlhumdev.2016.08.006

15. Lavallee A, Aita M, Bourbonnais A. Effectiveness of early interventions for parental sensitivity following preterm birth: a systematic review protocol. Syst Rev. 2017;6. https://doi.org/10.1186/s13643-017-0459-x

16. Premji SS, Currie G, Reilly S, et al. A qualitative study: mothers of late preterm infants relate their experiences of community-based care. PLOS ONE. 2017;12(3):e0174419. https://doi.org/10.1371/journal. pone.0174419

17. Fineout-Overholt E, Melnyk B, Schultz A. Transforming health care from the inside out: advancing evidence-based practice in the 21 st century. J Prof Nurs. 2005;21(6):335. https://doi.org/10.1016/ j.profnurs.2005.10.005

18. Premji SS, McNeil DA, Scotland J. Regional neonatal oral feeding protocol: changing the ethos of feeding preterm infants. $J$ Perinat Neonatal Nurs. 2004;18(4):371-384. https://doi.org/10.1097/ 00005237-200410000-00008

19. Smith DE. Institutional Ethnography: A Sociology for People. Walnut Creek, CA: AltaMira Press; 2005.

20. Rankin JM. Conducting analysis in institutional ethnography: analytical work prior to commencing data collection. Int J Qual Methods. 2017;16(1):1-9. https://doi.org/10.1177/1609406917734484

21. Smith DE. Institutional Ethnography as Practice. Lanham, MD: Rowman \& Littlefield; 2006.

22. Campbell ML. Institutional ethnography (IE), Texts and the materiality of the social. Paper presented at: System Sciences (HICSS), 47th Hawaii International Conference, 2014

23. Rankin JM. Conducting analysis in institutional ethnography: guidance and cautions. Int J Qual Methods. 2017;16(1):1-11. https://doi.org/ $10.1177 / 1609406917734472$
24. Smith DE. The Everyday World as Problematic: A Feminist Sociology. University of Toronto Press; 1987.

25. Warner BB, Sapsford A. Misappropriated human milk: fantasy, fear, and fact regarding infectious risk. Newborn Infant Nurs Rev. 2004;4(1):5661. https://doi.org/10.1053/j.nainr.2003.12.006

26. Benzies KM, Shah V, Aziz K, Lodha A, Misfeldt R. The health care system is making 'too much noise' to provide family-centred care in neonatal intensive care units: perspectives of health care providers and hospital administrators. Intens Crit Care Nurs. 2018;50:44-53. https:// doi.org/10.1016/j.iccn.2018.05.001

\section{About the Authors}

Catherine Ringham, PhD, BSN, RN, CNeoN(C), is a CIHR Health Systems Impact Postdoctoral Fellow. Her current work is exploring the social organization of Family Integrated Care (FICare) in Level 2 NICUs in Alberta and the effective and sustainable implementation of FICare.

Janet Rankin, $P h D, R N$, is an associate professor at the University of Calgary with a keen interest in institutional ethnographic exploration of health systems organization.

Lenora Marcellus, PhD, RN, is an associate professor at the University of Victoria School of Nursing. She has been involved in maternal/infant health most of her career as an educator and researcher.

For further information, please contact:

Catherine Ringham, $\mathrm{PhD}, \mathrm{BSN}, \mathrm{RN}, \mathrm{CNeoN}(\mathrm{C})$

University of Calgary and Alberta Health Services

University of Calgary

2500 University Drive NW

Calgary, AB T2N $1 \mathrm{~N} 4$

Canada

E-mail: clringha@ucalgary.ca

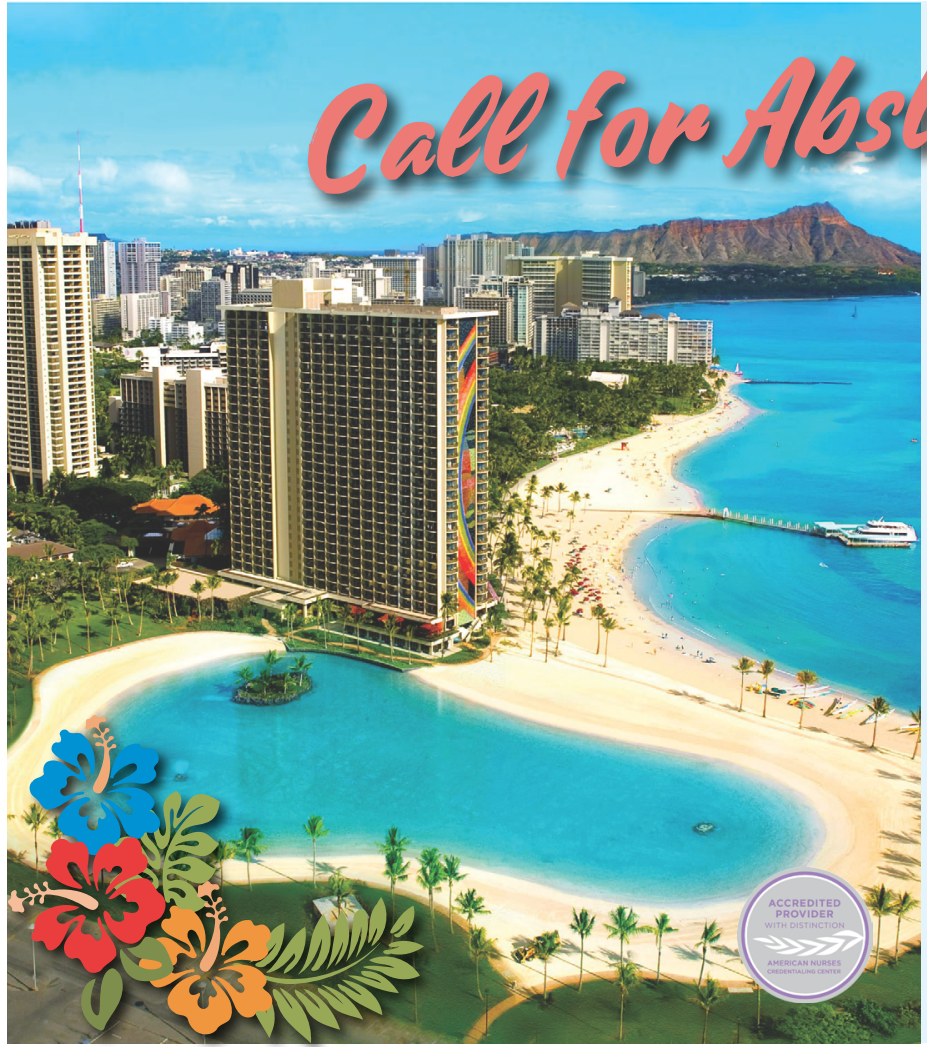

\section{Aprill 21-24, 2021}

\section{Spring 2021}

National Advanced Practice and

Low Risk Neonatal Nurses

Conferences

\author{
Hilton Hawaiian Village \\ Waikiki Beach, Honolulu, Hawaii
}
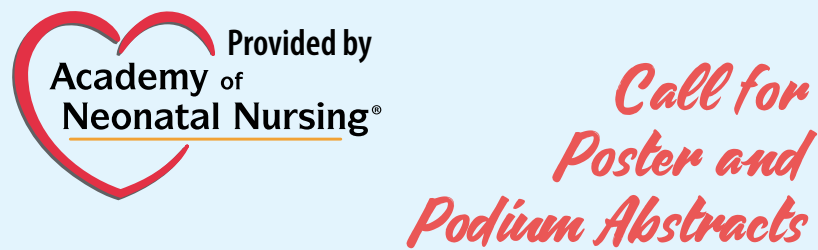

Submission Deadline January 31, 2021 